\title{
Multiple Metastases of a Transmissible Venereal Tumor in a Dog
}

\author{
Leonardo Schuler Faccini', Wilson Maccarini Legramanti', Lucas Teixeira de Castro', Ana Carolina Barreto Coelho', \\ Mariana Caetano Teixeira', Ana Lucia Shild ${ }^{2}$ \& Clairton Marcolongo Pereira ${ }^{3}$
}

\begin{abstract}
Background: Transmissible venereal tumor (TVT) is a common contagious neoplasm in dogs that spreads through coitus. Extra-genital presentations of this tumor are frequent and usually develop through implantation of neoplastic cells on exposed mucosae. TVT metastasis is rare, and when it happens it's usually affecting regional lymph nodes and adjacent cutaneous tissue.

Case: A female mixed breed dog, with estimated age of 7 to 11 months old, was rescued from the streets and taken to a veterinary clinic in the city of Porto Alegre, RS. The animal had multiple nodules on its body, vulva, ocular mucosa, and gingiva, along with signs of malnutrition and apathy. Cytological examination of the nodules and vulva was done and yielded a cytologic picture compatible with TVT. Weakly treatment with $0,3 \mathrm{mg} / \mathrm{m}^{2}$ vincristine sulphate was used until clinical cure was noted. Approximately two weeks after clinical cure, the dog showed a blue colored eye and was referred for ophthalmological, where it was diagnosed with vision loss due to glaucoma secondary to a neoplasm. The eye was then removed and sent for histopathological evaluation. Histopathology of the eye was compatible with TVT diagnosis. One month after enucleation the animal display dispenia, pain, aggressiveness and epistaxis. The animal was euthanized and submitted for post-mortem evaluation. At necropsy there was a well-defined grayish-white, nodule near the thalamus. Similar nodules were also found on the lung, and anterior chamber of the eye. Histologically, all the nodules were compatible with TVT. Immunohistochemical examination was done, with the neoplastic cells being positive for vimentin and negative for cytokeratin, CD79a, CD3 and CD117. Based on the post-mortem examination and clinical history, diagnosis of TVT was given.

Discussion: The clinical manifestation of the tumor in the genitalia presented by the animal is characteristic of TVT, but the extragenital presentation is less common. Although extragenital manifestations are well reported, most are due to auto-implantation (contact with the dog's own genitalia) or hetero-implantation (contact with the genitalia of another dog). Metastases originating from the genitalia are markedly less common (5\% of cases), and when they occur, they usually affect regional lymph nodes due to lymphatic communication. However, they can also occur in other organs, such as the liver, kidneys, spleen, tonsils, skin and subcutaneous tissue, bone, CNS, mesentery, and eyes. In this case, it is difficult to determine if the ocular tumor reported was due to metastasis or implantation by direct eye contact with TVT cells, since the animal lived on the street, and it was not possible to establish a more accurate history. The dog in this study was treated with vincristine sulfate; however, the treatment was stopped after one month, when no signs of the tumor were observed. Treatment with vincristine is the method of choice for TVT, since it is highly effective. However, rare cases of recurrence after treatment may occur. In addition, this drug does not cross the blood-brain barrier, and this may have favored the rapid reappearance of the tumor after treatment. The metastases from TVT to the CNS are extremely rare, with only a few cases reported in the literature. However, the possibility of metastases in animals with neurological and historical signs of TVT should be considered in the diagnosis. This case draws attention to the occurrence of TVT in the CNS of dogs with ocular TVT.
\end{abstract}

Keywords: neoplasm, transmissible venereal tumor, brain metastasis. 


\section{INTRODUCTION}

Canine transmissible venereal tumor (TVT) is a contagious, sexually transmitted neoplasm that develops in dogs and propagates through coitus [4].

Unlike other infectious tumors, TVT is not caused by an infectious agent, but rather by the allogeneic transference of tumor cells. It is estimated that this neoplasm originated from a mutation that occurred approximately 11,000 years ago [11]. The disease has spread and has been identified globally and is most common in regions with a greater number of stray dogs [11].

Although the primary manifestation is in the genital system, extragenital cases have been observed involving oral and nasal tissue, including the conjunctival mucosa adjacent to these tissues $[3,10]$ and less commonly, the eyes and central nervous system (CNS) [3]. Macroscopically, the lesions are friable and often hemorrhagic due to the low cohesion between the neoplastic cells; therefore, the first clinical sign observed tends to be bleeding [3]. TVT has a microscopic appearance like other round cell tumors, and the diagnosis is usually based on history, clinical signs, cytology, and histopathology. Immunohistochemistry has been used to differentiate this neoplasm from other round cell tumors [3]. The most common treatment is vincristine chemotherapy [3]. The objective of this report is to describe the clinical and pathological aspects of a case of TVT metastasis in the CNS of a dog.

\section{CASE}

A female mixed breed dog, approximately 9-month-old, was examined at a veterinary clinic in the city of Porto Alegre, RS. The animal had multiple nodules on its body, vulva, ocular mucosa, and gingiva, along with signs of malnutrition and apathy. Samples for cytological examination were collected through fine needle aspiration of the cutaneous nodules and a vaginal swab.

Cytological examination of the cutaneous tumors revealed a large number of round, moderately pleomorphic, isolated cells distributed in a layer, which stained poorly blue. The cytoplasm of the cells was vacuolated, and the nucleus: cytoplasm ratio was moderate. The nuclei were large and rounded, with loose chromatin. The nucleoli were rounded and prominent. There were one to two figures of mitosis per field. On the vaginal cytology, the same cell type was observed, in addition to a few inflammatory cells. The diagnosis was round cell neoplasia, with a cytologic picture compatible with TVT.
The animal was treated with vincristine sulphate $\left(0.3 \mathrm{mg} / \mathrm{m}^{2}\right.$ weakly $)$ and showed clinical improvement after one month of treatment. Approximately two weeks after clinical cure, the dog demonstrated a blue eye and was referred for an ophthalmological consultation. Diagnosed with vision loss due to glaucoma secondary to a neoplasm, the eye was removed and sent for histopathological evaluation.

On histopathological examination of the eye, neoplastic proliferation was observed in the iris, the ciliary body, and a focal area of the cornea. The cells were arranged in a layer, interspersed by a discrete fibrovascular stroma. The cells were rounded and had eosinophilic cytoplasm, round nuclei with condensed dotted chromatin, and a single evident nucleolus. Discrete anisocytosis, moderate anisokaryosis, and rare mitotic figures were observed. In addition, an extensive area of hemorrhage was seen in the posterior chamber, as well as a marked inflammatory infiltrate of degenerated neutrophils and hemorrhage in the ciliary body. In the cornea, there was moderate neovascularization, in addition to an extensive area of hemorrhage near Descemet's membrane, with multifocal areas of necrosis, an inflammatory infiltrate of degenerate neutrophils, and a marked deposition of fibrin. In the crystalline, rare Morgagni globules were observed. The changes observed on the histopathological examination were compatible with a diagnosis of TVT.

One month after enucleation, the animal displayed respiratory difficulty, pain, aggression, and epistaxis. Due to the worsening of clinical signs, the animal was euthanized and submitted for necropsy and histological evaluation.

At necropsy, there was a gray-white, well-defined, un-encapsulated nodule measuring $1.0 \mathrm{~cm}$ in diameter in the region of the optic chiasm (Figure 1A) and thalamus (Figure 1B). In the lung, there were several nodules of similar appearance (Figure 1C). In the anterior chamber of the eye, a small gray-white tumor was observed (Figure 1D). Histologically, the tumor cells were arranged in a layer and interspersed by a delicate fibrous stroma. The cells were predominantly rounded, although some were pleomorphic. The cytoplasm was scarce, finely granular, weakly basophilic, and had distinct limits (Figure 1E). The nuclei were yielding, prominent, and contained loose chromatin. There were one to two obvious nucleoli. Three mitotic figures were observed per field of higher magnifica- 
tion, in addition to a discrete inflammatory infiltrate of eosinophils, plasma cells, and lymphocytes. On immunohistochemical examination of the neoplastic tissue, the cells were observed to be positive for vimentin1 (Figure 1F) and negative for cytokeratin, CD79 1 , CD31, and CD1171.

\section{DISCUSSION}

The diagnosis of TVT was based on the clinical, cytological and histological changes observed. Immunohistochemical staining was also performed to aid in diagnosis and in the cell type observed in CNS and eye metastases. The results were positive
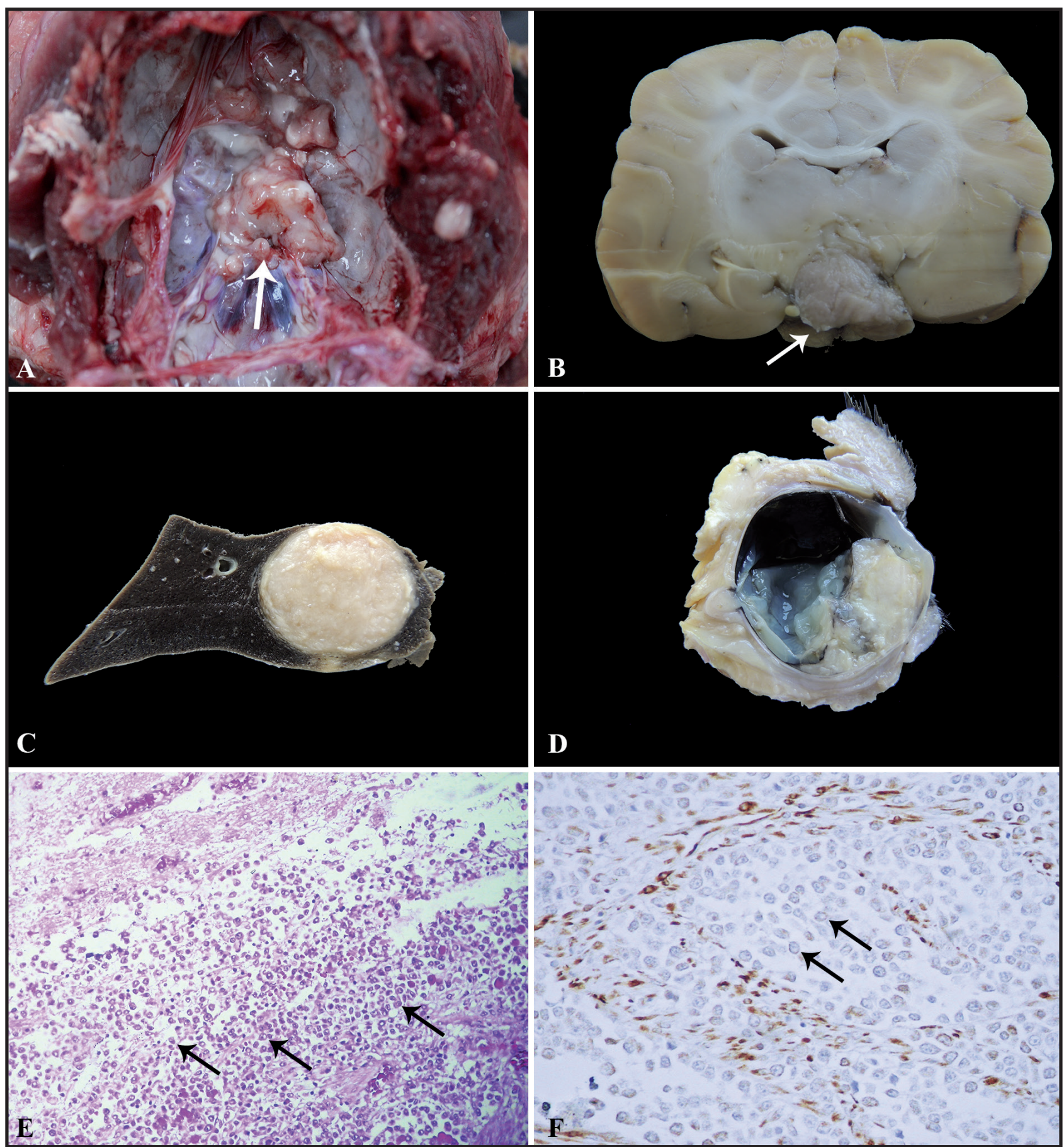

Figure 1. Metastatic Transmissible Venereal Tumor. A- Optic chiasm displaying a grayish white nodular mass measuring $1.0 \mathrm{~cm}$ in diameter. B- Ventral thalamus displaying a gray to white nodular mass measuring $1.0 \mathrm{~cm}$ in diameter. C- Lung displaying a mass like that of the central nervous system. D- Anterior chamber of the eye displaying a greyish-white mass. E- Neoplasm comprised of rows and cords of round to ovoid cells, with large, round nuclei [HE; Obj.20x]. F- Positive immunolabeling in the cytoplasm of the cells of the tumor for vimentin [Chromogen DAB]. 
for vimentin and negative for lymphocytes and mast cells, as observed in other TVT reports [6]. The clinical manifestation of the tumor in the genitalia presented by the animal is characteristic of TVT, but the extragenital presentation is less common. Although extragenital manifestations are well reported, most are due to auto-implantation (contact with the dog's own genitalia) or hetero-implantation (contact with the genitalia of another dog) [2]. Metastases originating from the genitalia are markedly less common (5\% of cases), and when they occur, they usually affect regional lymph nodes due to lymphatic communication. However, they can also occur in other organs, such as the liver, kidneys, spleen, tonsils, skin and subcutaneous tissue, bone, CNS, mesentery, and eyes [3]. In this case, it is difficult to determine if the ocular tumor reported was due to metastasis or implantation by direct eye contact with TVT cells, since the animal lived on the street, and it was not possible to establish a more accurate history. Primary ocular TVT cases have been reported [4]; however, most authors consider this manifestation as having a metastatic origin [4].

The dog in this study was treated with vincristine sulfate; however, the treatment was stopped after one month, when no signs of the tumor were observed. Treatment with vincristine is the method of choice for TVT, since it is highly effective. However, rare cases of recurrence after treatment may occur. In addition, this drug does not cross the blood-brain barrier [7] and this may have favored the rapid reappearance of the tumor after treatment, especially in the remaining eye. Probably because tumor proliferation was lodged in the optic nerve, in the region of the chiasm and in the CNS in the thalamus region and it may contribute for the metastasis for the eye. Animals with brain metastasis may present varying clinical signs, and the tumor may be in different regions, such as the pituitary [1,3], left piriform lobe, and optic chiasm [1]; the rostral and caudal portions of the left frontal lobe [3]; and the right frontal lobe [9]. The behavioral change observed in this case was likely due to the compression caused by the tumor, mainly in the thalamus region. Lesions in this region have been implicated in behavioral changes [1].

The metastases from TVT to the CNS are extremely rare, with only a few cases reported in the literature [3]. However, the possibility of metastases in animals with neurological and historical signs of TVT should be considered in the diagnosis. Immunohistochemistry is an important technique to aid in the determination of the cellular type present in the metastasis, aiding in the diagnosis of these cases.

\section{MANUFACTURER}

${ }^{1}$ DakoCytomation. Glostrup, Denmark.

Acknowledgments. This work was supported by grants from FAPES.

Declaration of interest. The authors report no conflicts of interest. The authors alone are responsible for the content and writing of the paper.

\section{REFERENCES}

1 Adams E.W. \& Slaughter L.J. 1970. A Canine Venereal Tumor with Metastasis to the Brain. Veterinary Pathology. 7(6): 498-502.

2 Esperidiao-Antonio V., Majeski-Colombo M., Toledo-Monteverde D., Moraes-Martins G., Fernandes J.J., Assis M.B. \& Siqueira-Batista R. 2008. Neurobiology of the emotions. Revista de Psiquiatria Clínica. 35(2): 55-65.

3 Ferreira A.J.A., Jaggy A., Varejäo A.P., Ferreira M.L.P., Correia J.M.J., Mulas J.M., Almeida O., Oliveira P. \&

Prada J. 2000. Brain and ocular metastases from a Transmissible Venereal Tumour in a dog. Journal of Small Animal Practice. 41(4): 165-168.

4 Ganguly B., Das U. \& Das A.K. 2016. Canine transmissible venereal tumour: A review. Veterinary and Comparative Oncology. 14(1): 1-12.

5 Komnenou A.T., Thomas A.L.N., Kyriazis A.P., Poutahidis T. \& Papazoglou L.G. 2015. Ocular manifestations of canine transmissible venereal tumour: A retrospective study of 25 cases in Greece. Veterinary Record. 176(20): 523.

6 Manning P.J. \& Martin P.D. 1970. Metastasis of canine transmissible venereal tumor to the adenohypophysis. Veterinary Pathology. 7(2): 148-152.

7 Park M.S., Kim Y., Kang M.S., Oh S.Y., Cho D.Y., Shin N.S. \& Kim D.Y. 2006. Disseminated transmissible venereal tumor in a dog. Journal of Veterinary Diagnostic Investigation. 18(1): 130-133. 
8 Pinczowski P., Gimeno M., Aceña C., Villegas A., De Martino A. \& Luján L. 2015. Brain metastasis in a case of canine transmissible venereal tumor after a supposed successful treatment with vincristine sulfate. Acta Veterinaria Beograd. 65(1): 137-142.

9 Placke M.E., Hill D.L. \& Yang T.J. 1987. Cranial Metastasis of Canine Transmissible Venereal Sarcoma. Journal of Veterinary Medicine Series A. 34(1-10): 125-132.

10 Rodrigues G.N., Alessi A.C. \& Laus J.L. 2001. Intraocular Transmissible Venereal Tumor in a Dog. Ciência Rural. 31(1): 141-143.

11 Strakova A. \& Murchison E.P. 2015. The cancer which survived: Insights from the genome of an 11000 year-old cancer. Current Opinion in Genetics and Development. 30: 49-55. 